\section{Aufnahme ärztlicher Komplementärmedizin in die Grundversicherung}

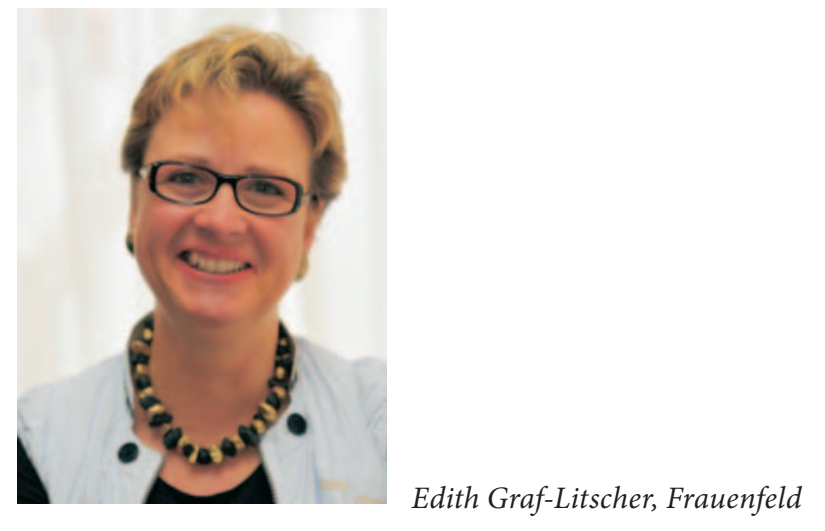

Die ärztliche Komplementärmedizin soll trotz klarem Volksentscheid nicht in den Leistungskatalog der Grundversicherung aufgenommen werden: Dies empfiehlt eine Fachkommission des Bundes, ohne Expertenwissen beizuziehen, und trotz mehrerer Verstösse gegen das eigene Geschäftsreglement. Ein Fall für die Geschäftsprüfungskommission.

Der Verfassungsartikel für Komplementärmedizin wurde am 17. Mai 2010 mit 67\% Ja-Stimmen überaus deutlich angenommen. Eine der Kernforderungen der Abstimmungskampagne war die Wiederaufnahme der ärztlichen Komplementärmedizin in die Grundversicherung. Es handelt sich dabei um folgende Methoden: Anthroposophische Medizin, Klassische Homöopathie, Neuraltherapie, Pflanzenheilkunde und Traditionelle Chinesische Medizin (TCM). Die entsprechenden Ärztegesellschaften haben im April 2010 dem Bundesamt für Gesundheit (BAG) Anträge zur Wiederaufnahme in die Grundversicherung mit detaillierten Fakten zu den gestellten Kriterien Wirksamkeit, Zweckmässigkeit und Wirtschaftlichkeit eingereicht.

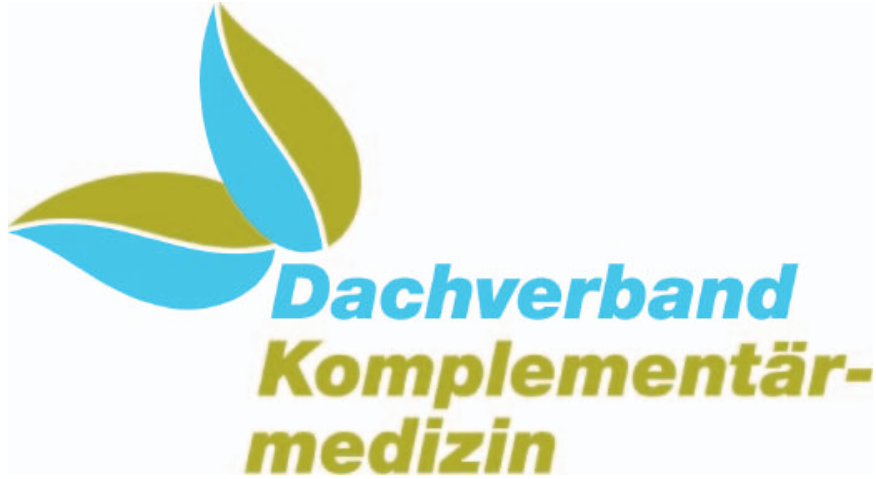

Die für die Prüfung zuständige Eidgenössische Kommission für allgemeine Leistungen und Grundsatzfragen (ELGK) hat am 7. Dezember 2010 mitgeteilt, dass sie dem Bundesrat empfiehlt, keine der fünf komplementärmedizinischen Methoden in den Leistungskatalog der obligatorischen Krankenpflegeversicherung aufzunehmen. Bei der genaueren Betrachtung der Arbeitsweise dieser Kommission haben wir festgestellt, dass Zweifel an der korrekten Arbeitsweise und damit auch an der Empfehlung der ELGK angebracht sind. So verstösst die ELGK mehrfach gegen die eigene Geschäftsordnung: Befangene Krankenkassenvertreter treten nicht in den Ausstand, obwohl die Krankenkassen mit Komplementärmedizin in der Zusatzversicherung grosse Gewinne machen. Auch werden keine externen Experten beigezogen, obwohl dies die Geschäftsordnung explizit vorschreibt. Das BAG hat im neuen «Handbuch» zwar verpflichtende Kriterien zur Einreichung der Anträge ärztlicher Komplementärmedizin, jedoch keine Kriterien für die objektive, sachliche und nachvollziehbare Prüfung der Anträge festgelegt. Damit drohen die Anträge erneut einem Willkür-Entscheid zum Opfer zu fallen.

Die Entscheidungsfindung der Leistungskommission ist sehr intransparent und wenig vertrauensbildend. Eine überparteiliche Parlamentariergruppe hat deshalb der Geschäftsprüfungskommission des Nationalrates den Antrag gestellt, eine Untersuchung zum Entscheidungsprozess der ELGK einzuleiten. Ich bin froh, wenn sich die Geschäftsprüfungskommission der Sache annimmt und ein neutrales Urteil fällt.
Donnerstag, 17. März 2011 (Nachmittag); Bern

Donnerstag, 5. Mai 2011 (Vormittag); Bern

Donnerstag, 5. Mai 2011 (Nachmittag); Bern

Donnerstag, 30. Juni 2011 (Nachmittag); Bern 
Wir kennen nur das Urteil der Leistungskommission, nicht aber die Begründungen. Die Kommission hat sich ins Abseits manövriert, weil zahlreiche Verfahrensregeln missachtet wurden.

Der Entscheid über die Aufnahme der ärztlichen Leistungen der Komplementärmedizin liegt nun beim Gesundheitsminister, Didier Burkhalter. Er muss sich nicht an die Empfehlungen seiner Kommission halten, sondern prüfen, ob die Anträge die rechtlichen Vorgaben erfüllen. Auch muss er berücksichtigen, dass das Volk komplementärmedizinische Leistungen in der Grundversicherung will. Die parlamentarische Gruppe Komplementärmedizin empfiehlt dem Gesundheitsminister, sich nicht auf den Antrag der Leistungskommission zu stützen und eigene, unabhängige Experten einzusetzen, die ihn bei der Wie- deraufnahme der Leistungen in die Grundversicherung beraten. Der Gesundheitsminister ist der Verfassung und den Gesetzen verpflichtet, nicht aber den Anträgen von beratenden Kommissionen.

Edith Graf-Litscher, Nationalrätin (SP TG) und Co-Präsidentin Dachverband Komplementärmedizin

\section{Kontakt}

Dachverband Komplementärmedizin

Amthausgasse 18

3011 Bern, Schweiz

Tel. +41315600024

info@dakomed.ch

www.dakomed.ch

Postkonto 90-151274-4

\section{Positiver Entscheid des EDI zu den komplementärmedizinischen Anträgen}

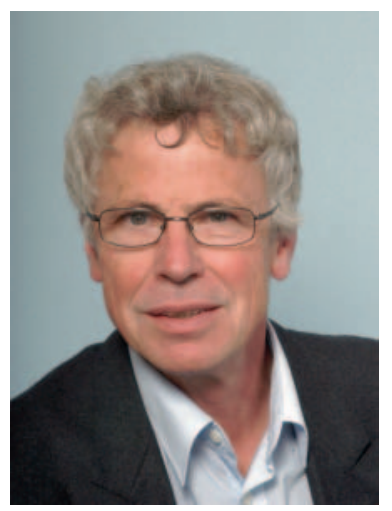

Das Eidgenössische Departement des Innern (EDI) hat am 11. Januar 2011 entschieden, die Leistungspflicht der obligatorischen Krankenpflegeversicherung für die fünf fachärztlichen Behandlungsmethoden Anthroposophische Medizin, Klassische Homöopathie, Neuraltherapie nach Huneke, Phytotherapie und Traditionelle Chinesische Medizin für eine befristete Zeit von Januar 2012 bis Ende 2017 wieder einzuführen. Die Übergangsperiode soll genutzt werden, um kontroverse Aspekte zu klären und Klarheit über die Wirksamkeit, Zweckmässigkeit und Wirtschaftlichkeit (WZW-Kriterien) zu schaffen. Die Wirksamkeit der fünf Methoden soll überdies von einer international anerkannten Institution beurteilt werden. Das Departement beauftragt die Eidgenössische Kommission für allgemeine Leistungen und Grundsatzfragen (ELGK) in einer neuen Zusammensetzung, dem Departement im Laufe des Jahres 2016 eine neue Empfehlung zu unterbreiten.

Die Union schweizerischer komplementärmedizinischer Ärzteorganisationen ist erfreut über den Entscheid des Bundesrates Didier Burkhalter. Der Gesundheitsminister trägt damit sowohl dem Volkswillen als auch dem Krankenversicherungsgesetz Rechnung. Die Wirtschaftlichkeit der ärztlichen Komplementärmedizin ist mittlerweile gut dokumentiert und wurde sowohl von der santésuisse als auch von der FMH bestätigt. Die anhaltende Kontroverse zum Wirksamkeitsnachweis kann überwunden werden, wenn sich Antragsteller und Beurteilungsinstanzen $\mathrm{zu}$ verbindlichen methodischen Vorgaben zusammenfinden. Die Union ist überzeugt, dass bis im Jahr 2015 die Unklarheiten beseitigt und die Lücken im Nachweis der WZW-Kriterien geschlossen werden können.

Dr. med. Hansueli Albonico

Präsident schweizerischer komplementärmedizinischer Ärzteorganisationen

(Anmerkung: Der Entscheid des EDI wurde nach Redaktionsschluss dieser Ausgabe der Schweizerischen ZEITSCHRIFT FÜR GANZHEITSMEDIZIN gefällt.) 